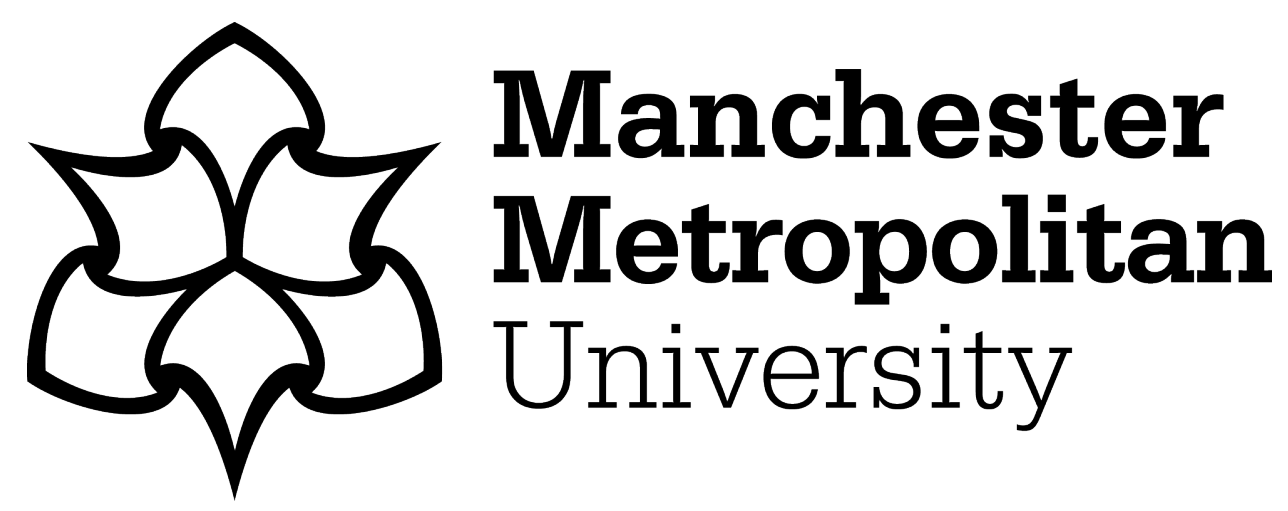

Usman Sarwar, Muhammad, Rehman Javed, Abdul, Kulsoom, Farzana, Khan, Suleman, Tariq, Usman and Kashif Bashir, Ali (2021) PARCIV: Recognizing physical activities having complex interclass variations using semantic data of smartphone. Software: Practice and Experience, 51 (3). pp. 532549. ISSN 0038-0644

Downloaded from: https://e-space.mmu.ac.uk/625900/

Version: Accepted Version

Publisher: Wiley

DOI: https://doi.org/10.1002/spe.2846

Please cite the published version 


\title{
PARCIV: Recognizing Physical Activities having Complex Inter-class Variations using Semantic Data of Smartphone
}

\author{
Muhammad Usman Sarwar ${ }^{1 *}$ | Abdul Rehman Javed ${ }^{2 *}$ | \\ Farzana Kulsoom $^{3 \ddagger}$ ｜ Suleman Khan ${ }^{3 \Phi ~ \mid ~ U s m a n ~ T a r i q ~}{ }^{4 \S}$ \\ | Ali Kashif Bashir ${ }^{\ddagger}$
}

${ }^{1}$ Department of Computer Sciences, Air University and ASET: Ambient Systems and Emerging Technologies Lab, Islamabad 44000, Pakistan

${ }^{2}$ National Center for Cyber Security, Air University and ASET: Ambient Systems and Emerging Technologies Lab, Islamabad 44000, Pakistan

${ }^{3}$ Department of Electrical Computer and Biomedical Engineering, University of Pavia, Pavia, Itlay

${ }^{4}$ College of Computer Science and Engineering Prince Sattam bin Abdulaziz University, Saudi Arabia

${ }^{5}$ School of Computing, Mathematics, and Digital Technology, Manchester Metropolitan University, United Kingdom

Correspondence

Abdul Rehman Javed, National Center for Cyber Security (NCCS), Air University, Islamabad, Pakistan

Email: abdurrahman.j74@gmail.com

Funding information

This work received no external funding.
Smartphones are equipped with precise hardware sensors including accelerometer, gyroscope, and magnetometer. These devices provide real-time semantic data that can be used to recognize daily life physical activities for personalized smart health assessment. Existing studies focus on the recognition of simple physical activities but they lacked in providing accurate recognition of physical activities having complex inter-class variations. Therefore, this research focuses on the accurate recognition of physical activities having complex inter-class variations. We propose a two-layered approach called PARCIV that first clusters similar activities based on semantic data and then recognize them using a machine learning classifier. Our two-layered approach first bound the highly indistinguishable activities in clusters to avoid misclassification with other distinguishable activities and thereafter recognize them on a fine-grained level within each cluster. To evaluate our approach, we make an android application that collects labeled data by using smartphone sensors from ten participants, while performing activities. PARCIV recognizes distinguishable as well as indis- 
tinguishable activities with high accuracy of $99 \%$ on the self-collected dataset. Furthermore, PARCIV achieve 95\% accuracy on the publicly available dataset used by state-ofthe-art studies. PARCIV outperforms various state-of-theart studies by $8-17 \%$ for simple activities as well as complex activities.

\section{KEYWORDS}

Semantic Data, Healthcare, Smartphone Sensors, Accelerometer, Complex Confused Activities, Inter-class Variations

\section{1 | INTRODUCTION}

Healthcare is enriched with semantic data representing information about the personal health of an individual. Semantic data depicts the relationships among data (i.e data obtained from smartphone sensors for activities such as fall detection and activity recognition). Despite such richness in the healthcare domain, not all the Machine Learning $(\mathrm{ML})$ techniques in automated healthcare systems can work efficiently on semantic data. Right now, among the best generally utilized $M L$ techniques for experimentation of semantic data are Support Vector Machine (SVM), Artificial Neural Network (ANN), and Random Forest (RF). Semantic data collected by smartphone sensors (accelerometer, gyroscope, and magnetometer) of daily life activities shares some common semantic information and characteristics about physical health assessment [1].

In automated healthcare systems, physical activity recognition is a challenging problem due to its practical applications like healthcare support, smart sports, elder-care and assisted living [2]. Physical activity recognition and assessment have a direct connection with an individual's health and physical fitness. Physical activity recognition improves the individual's health by monitoring and analyzing activities of daily life. In healthcare, doctors can examine the health conditions of individuals according to their performed activities [1]. In today's busy schedule, people want a healthy lifestyle. They pay more attention to their health, physical fitness, the suggestion and recommendations provided by the care providers [1 2 2 3,4$]$. Physical inactivity is rising as a big issue nowadays. Several individuals face serious diseases due to a lack of physical activities. Authors in [5] stated that it is the $4^{\text {th }}$ leading risk factor for people. Also, blood pressure and obesity are quietly closed to physical inactivity [6]. Authors showed that physical fitness can decrease mental disorder, cancer, diabetes, muscle issues, weight issues, emotional issues, and depression as well.

Physical activity recognition was first initiated using on-body sensors. On-body Sensors were used back in 2004 by the study [7]. They placed bi-axial sensors at different body positions to detect 20 physical activities. The challenge in this scenario is to wear on-body sensors and carry them all the time. In state-of-the-art, smartphones have been extensively used for physical activity recognition [8, 1]. Smartphones embedded with various precise sensors (i.e., accelerometer, gyroscope and magnetometer) has replaced wearable devices due to its unobtrusive nature. It is easy to carry and today everyone has a smartphone. The availability of the smartphone is the biggest breakthrough in a computing environment. Smartphones are the most ubiquitous, non-obtrusive, reliable and beneficial source to recognize physical activities and other health-related issues (i.e., calories burned, heart rate monitoring) [9].

Many researchers reported that the accelerometer sensor is the most reliable and cheapest alternate of wearable sensors for physical activity recognition. Authors in [7, 8, 10,11] analyzed that accelerometer can be used in combination with other sensors like gyroscope, light, proximity, barometer, linear acceleration and magnetometer for 
better activity recognition. Furthermore, there is a large increase in the inventions of daily monitoring systems that can detect the individual's health, lifestyle, activities, behavior, and emotions.

Existing work on physical activity recognition mainly focused on six basic activities: walking, standing, sitting, running, upstairs and downstairs which are quite normal daily life activities and can be recognized easily. While other complex activities have less discriminating information as they are very similar in behavior and pattern (e.g., upstairs with downstairs, cycling with upstairs, fast running with jogging, laying positions and sitting positions). Therefore these activities get confused with each other in the recognition process and hence results in misclassification.

To address the above limitations, we make the following contributions.

- Propose a two-layered approach named PARCIV for physical activity recognition having Complex Inter-class variations.

- PARCIV first clusters the activities having complex inter-class variations based on semantic data and then recognize them on a fine-grained level within each cluster.

- Provide pattern analysis of 15 types of complex physical activities.

- Evaluate PARCIV on a self collect dataset from 10 participants and publically available datasets.

- PARCIV achieved promising results on the self-collected dataset as well as on publically available datasets used in state-of-the-art studies.

The remainder of the paper is structured as follows: Section 2 presents the related work on physical activity recognition, Section 3 describes the proposed approach for physical activity recognition and Section 4 shows the experimental setup containing evaluation measures, activity representation analysis, dataset, comparisons of results and discussion. Section 5 concludes the paper and shows the future direction.

\section{2 | LITERATURE REVIEW}

In this section, we present the related studies focusing on physical activities using streaming data collected either by smartphone or on-body sensors as well as other studies related to healthcare. In healthcare applications, the use of semantic data may extraordinarily improve the worthiness of data by using machine learning methods 12,13 14 15]. Healthcare data collected by smartphone sensors of activities of daily life share some common semantic information and characteristics. A smartphone is equipped with precise sensors like Motion sensors, Environmental sensors, Position sensors. Motion sensors such as accelerometer, gyroscope, and magnetometer are the most valuable and well-studied sensors for physical activity recognition. The study [7] presents an approach which identified physical activities with the bi-axial accelerometer on 5 body positions: hip, wrist, upper arm, ankle, and thigh. They used Decision Tree (DT) C4.5 and Naive Bayes (NB) ML techniques for classification. After applying the ML techniques, they show that the data collected from the thigh position shows higher accuracy than the data of all other positions. Some other studies [7 8 8 11] used a similar approach for physical activity recognition. The study [16] collected data from 29 individuals of an educational institute using an android application at the sampling rate of 20 samples per second $(20 / \mathrm{sec})$. The smartphone was placed in the participant's pocket. They selected a static window of 200 samples to apply the predictive models. They applied three classification methods: DT (j48), Logistic Regression (LR) and Multilayer Perceptron (MLP) using ten-fold-cross-validation.

Some studies also preferred dynamic window size over static window size for classification process [17, 18, 19]. They collected data at different frequencies. The study [17] shows that the data collected at different frequencies 
can positively affect the overall performance of activity recognition as well as the energy consumption of the battery. They collected data at different frequencies (i.e. $5 \mathrm{hz}, 16 \mathrm{hz}, 50 \mathrm{hz}$, and $100 \mathrm{hz}$ ) and saved battery energy by $50 \%$. A similar study [18 proposed a low energy physical activity recognition system using a smartphone. They used the discrete variables that were obtained from the accelerometer sensor. A discretization process was used for each variable that drains very little energy. The approach determined the performed activity and the frequency at which it is performed. Furthermore, the technique saved a lot of battery power up to 27 hours while maintaining accuracy. Data were collected from 10 users with different smartphones. Ameva algorithm was used to help the new algorithm in the discretization process.

The study 20 brings some changes in the behavior of getting data from the accelerometer and applying $\mathrm{ML}$ techniques on it. First, they divide the data collected from a tri-axial accelerometer into short non-overlapping windows and then convert each window into a feature vector. For supervised learning, they treated each instance as an independent identical distributed training instance. A multi-scale ensemble method applied for activity recognition as some activities have repetitive behavior. However, their experiments show low results for upstairs, lying, downstairs, walk and jogging activities. The study 10 provides a comprehensive survey on human activity with all available sensors in the smartphone. These sensors include accelerometer, ambient temperature, gyroscope, linear acceleration, magnetometer, barometer and proximity sensor. The study [8] introduced a framework to recognize physical activities. The framework collects accelerometer data from 10 participant's smartphones with a rate of 50 samples per second (50/sec). They used a fixed size window approach to segment the data. A total of 100 sample window was selected to apply the ML algorithm. They analyzed, when and how to use sensors individually or in combination. They choose five body positions right pocket, left the pocket, on the belt, right upper arm, and right wrist. They investigate the performance of the data collected from single and multiple participants.

The research work [11] analyzed that the motion recognition is a difficult step to recognize or even it can be worse if the motion is showing repetitions and abnormal behavior like walking and fast walking, sharp turn upstairs and downstairs. Authors combined the latest positioning technologies and sensors to capture human movements in natural environments. They investigated some common motion states like standing, sitting, walking, lying, fast walking, U-Turn, and sharp turn. They observed that the static states like sitting, lying, and standing are easy to recognize, but dynamic states like U-Turn and sharp turn are difficult to recognize. Similarly, the study [21] worked on activity recognition by placing the smartphone in different positions and orientations. They used smartphone sensors like accelerometer, gyroscope, proximity, light, and magnetometer sensor. Data were analyzed in a sense either the smartphone was in the participant's pants pocket or shirt's pocket or hand by using light and proximity sensors. They used decision tree j48, naive bayes and support vector machine for activity recognition.

By considering the effect of flexibility in orientation of smartphone, placement of smartphone and participant's variations, the study [22], proposed an approach based on the Coordinate Transformation and PCA. For decreased performance due to the inherent difference in signal properties of different types of placement, they used Online SVM. The study [23] highlights that the accurate recognition of activities depends on the efficient feature selection method from the time series data. They proposed an approach for dynamic feature extraction and then the results of dynamic and static features were compared. They analyzed that the Convolutional Neural Networks (CNN) provides a better recognition rate with dynamic features rather than other common ML algorithms (i.e., MLP, SVM, and k-nearest neighbor(KNN)).

Authors in 24 first extracted features (mean, median, auto-regressive coefficients, etc.) from the raw dataset. They applied Linear Discriminant Analysis (LDA) and Kernel Principal Component Analysis (KPCA) to make these features robust. They trained the Deep Belief Network (DBN) for recognition of activities. The comparison was shown with the traditional approaches: SVM and ANN. An unsupervised approach was proposed by [2] to classify physical 
activities using the smartphone accelerometer sensor. They gathered data on normal life exercises and made an undirected graph utilizing Euclidean Distance (ED). This diagram was then passed to the MCODE clustering algorithm to group daily life activities.

An approach [25], recognize daily routine activities by classifying semantic data on the remote server. They used one smartphone having an accelerometer to collect data at the frequency of 100 samples per second and used a static window technique to segment the data. The smartphone was placed in the subject's pocket to gathered all the activities data. Later, they applied $10 \mathrm{ML}$ techniques on the data to recognize activities remotely. The study [26] presented a methodology for elderly people to assist them in their daily life activities. They used the Ameva algorithm to reduce battery consumption. They reported that the combination of the gyroscope, magnetometer, and barometer resulted in the improvement of the recognition rate.

To the best of our knowledge, existing physical activity recognition approaches either only consider simple activities (e.g. walking, running, standing, sitting and cycling) or have low recognition rate to recognize activities having complex inter-class variations (e.g. upstairs, downstairs, lying positions, cross-legs, jogging, and fast walking). Moreover, existing studies have not performed the pattern analysis information of complex activities. Table 1 presents an overview of which smartphone sensors, sampling frequency, sliding window and dataset used by state-of-the-art studies. Table 2 presents an overview of several individuals, smartphone sensors and sensors placing positions used by state-of-the-art studies.

TAB LE 1 Sensors and Datasets used in Related Work

\begin{tabular}{|c|c|c|c|c|c|c|c|c|c|c|c|c|c|c|c|c|}
\hline Type & - & 8] & 25] & 27] & 26] & 10] & 20] & 11] & 18] & 21] & $7,28]$ & 16] & 29] & $30]$ & 17] & 19] \\
\hline & Accelerometer & $\checkmark$ & $\checkmark$ & $\checkmark$ & $\checkmark$ & $\checkmark$ & $\checkmark$ & $\checkmark$ & $\checkmark$ & $\checkmark$ & $\checkmark$ & $\checkmark$ & $\checkmark$ & $\checkmark$ & $\checkmark$ & $\checkmark$ \\
\hline & Magnetometer & $\checkmark$ & - & - & - & $\checkmark$ & - & $\checkmark$ & - & $\checkmark$ & - & - & - & - & - & - \\
\hline & Light Sensor & - & - & - & - & $\checkmark$ & - & - & - & $\checkmark$ & - & - & - & - & - & - \\
\hline \multirow[t]{6}{*}{ Sensors } & Gyroscope & $\checkmark$ & - & - & - & $\checkmark$ & - & $\checkmark$ & - & $\checkmark$ & - & - & - & - & - & - \\
\hline & Proximity & - & - & - & - & $\checkmark$ & - & - & - & $\checkmark$ & - & - & - & - & - & - \\
\hline & Barometer & - & - & - & - & $\checkmark$ & - & - & - & - & - & - & - & - & - & - \\
\hline & Linear Accel. & $\checkmark$ & - & - & - & $\checkmark$ & - & - & - & - & - & - & - & - & - & - \\
\hline & $10-50$ & - & $\checkmark$ & - & - & - & $\checkmark$ & $\checkmark$ & - & $\checkmark$ & $V$ & $\checkmark$ & $\checkmark$ & $\checkmark$ & $\checkmark$ & $\checkmark$ \\
\hline & 100 & $\checkmark$ & - & - & - & - & - & - & - & - & - & - & - & - & $\checkmark$ & - \\
\hline Sampling & 125 & - & - & - & $\checkmark$ & - & - & - & - & - & - & - & - & - & - & - \\
\hline \multirow[t]{2}{*}{ Frequency } & 128 & - & - & $\checkmark$ & - & - & - & - & - & - & - & - & - & - & - & - \\
\hline & 200 & - & - & - & - & - & - & - & $\checkmark$ & - & - & - & - & - & - & - \\
\hline Sliding & - & - & - & - & - & - & - & - & $\checkmark$ & - & - & - & - & - & $\checkmark$ & $\checkmark$ \\
\hline \multicolumn{17}{|l|}{ Window } \\
\hline & Self & $\checkmark$ & $\checkmark$ & $\checkmark$ & $\checkmark$ & $\checkmark$ & $\checkmark$ & $\checkmark$ & $\checkmark$ & $\checkmark$ & $\checkmark$ & $\checkmark$ & $\checkmark$ & $\checkmark$ & $\checkmark$ & $\checkmark$ \\
\hline Dataset & Publicly & - & - & - & $\checkmark$ & - & - & - & - & - & - & - & - & - & - & - \\
\hline
\end{tabular}


TAB LE 2 Setup for Data Collection in Related Work

\begin{tabular}{|c|c|c|}
\hline Type & Setup & References \\
\hline \multirow[t]{2}{*}{ Individuals } & Single & 19] \\
\hline & Multiple & $8,25,27,26,20,18,7,7,16,29,28,30$ \\
\hline \multirow[t]{2}{*}{ Single Sensor } & Single Accelerometer & $25,27,20,18,7,16,28,30,19]$ \\
\hline & Multiple Accelerometer & $8,10,11,7]$ \\
\hline \multirow[t]{4}{*}{ Multiple Sensors } & Accelerometer, Magneto, Gyroscope, Linear Acceler & [8] \\
\hline & Acceler, Magneto, Light Sen, Proxi, Barome, Gyro, Linear Acc & 10 \\
\hline & Accelerometer, Magnetometer, Gyroscope & 11] \\
\hline & Accelerometer, Magnetometer, Light Senor, Proximity, Gyroscope & 21] \\
\hline \multirow[t]{3}{*}{ Sensor Position } & Pocket & $8,25,27,26,11,7,16$ \\
\hline & Arm, Knee, Waist, Pocket, Abdomen, Thigh & $20,7,28,30]$ \\
\hline & Hip & 18 \\
\hline
\end{tabular}

\section{3 | PROPOSED METHODOLOGY}

In this section, we explain our proposed approach named PARCIV: Physical Activity Recognition having Complex Inter-class Variations. Figure 1 summarizes our proposed approach. Below we describe algorithm description, data collection, sensing application, preprocessing, data balancing and classification models for activity recognition.

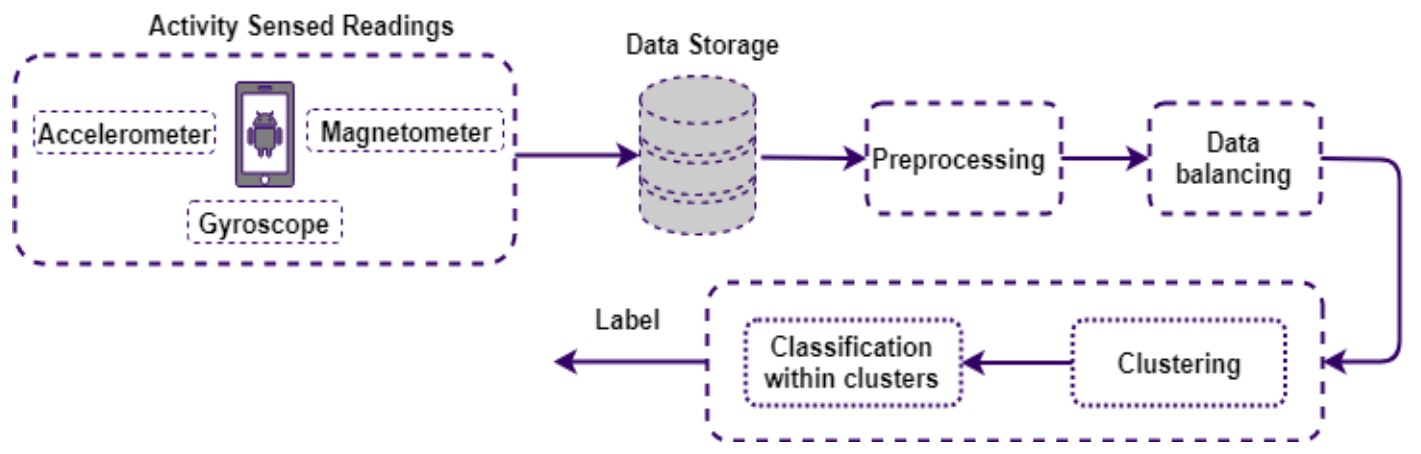

FIGURE 1 Block Diagram for the Proposed Approach

\section{1 | PARCIV Algorithm Description}

The feature matrix consisting of semantic data of activity instances $I_{j i}$ of activity $A_{i}$ is given to a ML model K-means to group the instances of similar activities into clusters $S_{u k}$. Each cluster $S_{u k}$ come into existence by minimizing the error objective function that measures the distance of each activity instance from their respective cluster center given 


$$
\sum_{u=1}^{U_{k}} \sum_{j=1}^{J}\left\|I_{j k}-C_{u k}\right\|^{2}
$$

where $C_{u} k$ is cluster center for each cluster $S_{u k}$. Then each instance $U K_{i}$ of each cluster $S_{u k}$ is given to classifier kNN which returns a label $L{ }^{A_{i}}$ on the basis of nearest neighbour.

$I$ : total number of activity classes;

$J:$ total number of activity instances per class;

$A_{i}: i^{\text {th }}$ activity class

$I_{j i}: j^{\text {th }}$ instance of activity $i^{\text {th }}$ class;

$S_{u k}: u^{\text {th }}$ clusters of activity $A_{i}$;

$U K_{i}: i^{\text {th }}$ instance of $u^{\text {th }}$ cluster;

$L^{A_{i}}$ : label of recognized activity class;

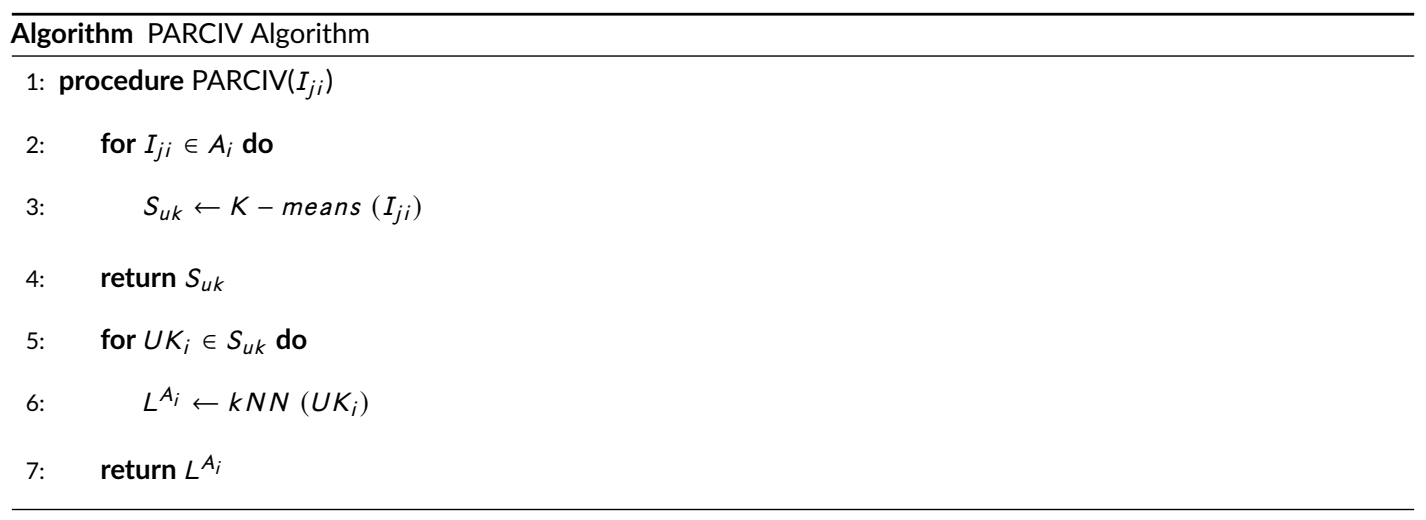

\subsection{Data Collection}

This section presents our data collection rules and protocols. We collect data from smartphones: Oppo F3 and Oppo F1s. To perform physical activities, we voluntarily recruited 10 participants (mean age $=25.0$ with a range of 21 to 30 years). Out of 10 participants, 9 were male and 1 was female. We ask participants to perform selected activities using a smartphone. While they perform activities, a smartphone was placed in the left pocket to capture the progress of the activity to its completion. This application permits us to control which sensor data (e.g., accelerometer, gyroscope, magnetometer) to collect and how frequently to collect. In all cases, we collected the data at a fixed frequency of 40 samples per second, so we had 2400 samples per minute. This frequency is fine enough to capture all required actions as recent studies show that the sensor invoking frequency is good between 10-50 samples per second [31. 18] in order save battery.

The data collection was supervised by our team members to ensure the quality of the data. Dataset consists of sensor events generated while performing the activities. Each activity was performed continuously for approximately 4-5 minutes except the upstairs and downstairs because it was difficult to gather data with the limited stairs and 
physical fitness of the participant. Not all participants performed each activity. Some participants performed ten activities and some performed 7. All the sensor readings were recorded in the comma-separated file (CSV). To ensure recorded reading are well organized we assigned a timestamp to each reading. In this way, our data set consists of 9 features and 1 label. For the ground truth data, we assigned labels manually to all the sensed readings as we have the ground truth information about the task being performed. Table 3 shows the characteristics of dataset. Illustration of activities posture is shown in Figure 2

TABLE 3 Characteristics of the Collected Dataset

\begin{tabular}{|c|c|}
\hline Dataset & Human Activity Recognition \\
\hline Number of Participants & 10 \\
\hline Mean Age & 26 \\
\hline Health Condition & Healthy \\
\hline Total Features & 10 \\
\hline Total Activities & 15 \\
\hline Dataset Property & Imbalance \\
\hline Activities of Daily Life & $\begin{array}{c}\text { Sitting Straight. } \\
\text { Sitting Left Leg Over Right Leg } \\
\text { Sitting Right Leg Over Left Leg } \\
\text { Sitting Cross leg } \\
\text { Walking with normal Speed } \\
\text { Walking Fast } \\
\text { Jogging } \\
\text { Running } \\
\text { Cycling } \\
\text { Standing } \\
\text { Upstairs } \\
\text { Downstairs } \\
\text { Laying Straight } \\
\text { Laying Over Left Side } \\
\text { Laying Over Right Side }\end{array}$ \\
\hline
\end{tabular}




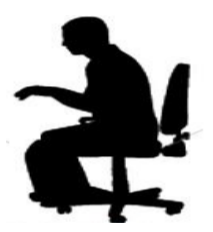

Sitting Straight
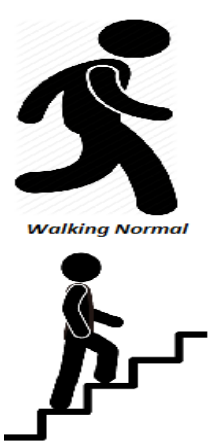

Upstairs

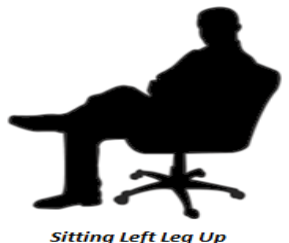

Sitting Left Leg Up
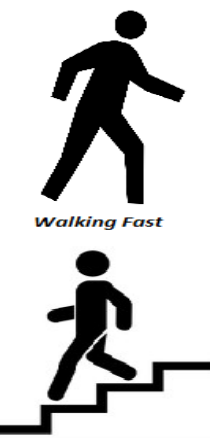

Downstairs
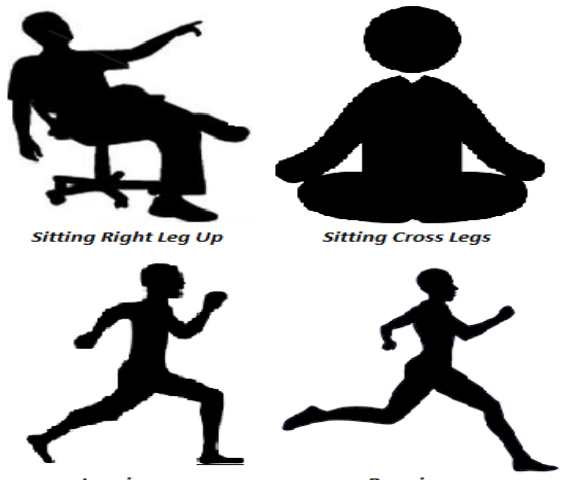

Running

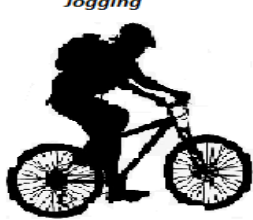

Cycling

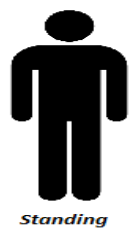

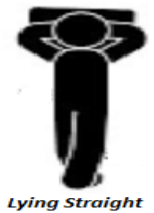

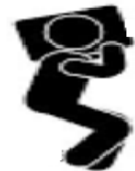

Lying Over Left side

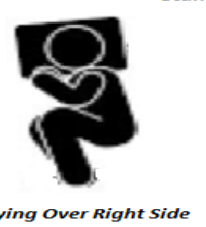

FIGURE 2 Graphical Representation of the Activities

\section{3 | Smart Phone Sensing Data Application}

In this work, we make an android application PARCIV, run-able for every type of Android smartphone. For android, our minimum operating system requirement is 4.0, which covers the majority of Android devices [32]. Android allows applications to read data from smartphone sensors, while iOS has a much stricter policy that only allows third-party apps to collect data from a very limited set of sensors.

- It consists of a graphical user interface with an edit-text to label the performed activity as ground truth.

- A service was made to run continuously in the background to collect data from all three sensors.

- Three smartphone sensors accelerometer, gyroscope, and magnetometer were used to perform the data collection task. Data were collected at a fixed frequency of 40 samples per second.

- A service was programmed to flush all the data into a Comma Separated File (CSV) file when the back key was pressed.

\section{4 | Data Preprocessing}

In our data collection task 3.2 as explained that the data was collected by ten participants while the smartphone was kept in participant's left pocket in most cases. Noisy samples are generated when a participant places the smartphone 
in the pocket and removes the smartphone from the pocket. It took almost 2-3 seconds, which means 120-200 samples at the start and the end. To get reasonable results, we remove this noisy data from each activity.

\section{5 | Data Balancing}

In the case of imbalanced class distribution, the machine learning model does not work well [33 34]. As studied in [33, SMOTE is used to increase the instances of the minority class. It creates a new instance of the minority class based on specified nearest neighbors of the minority class which improves the representation of the minority classes which results in improving the recognition performance of the $M L$ model. To make a new instance it calculates the distance between an original instance and the nearest neighbors. Then it multiplies the new distance with the range between $0-1$ and then it is added into the original instance thus a new instance comes into existence. Below we explain the example of generating synthetic instances.

Suppose a sample $(1,2)$ and let $(3,4)$ be its nearest neighbor. $(1,2)$ is the sample for which k-nearest neighbors are being identified. $(3,4)$ is one of its k-nearest neighbors.

Let:

$$
\begin{array}{ll}
D s 1_{1}=1, & D s 2_{1}=2 \\
D s 1_{2}=3, & D s 2_{2}=4
\end{array}
$$

The new samples will be generated as

$$
\left(D s 1^{\prime}, D s 2^{\prime}\right)=(1,2)+\operatorname{rand}(0-1) *(3-1,4-2)
$$

where rand(0-1) generates a random number between 0 and 1.

\section{6 | Machine Learning Model}

We applied two machine learning algorithms to cluster and classify the activities. Below are the algorithms:

- K-Means: K-means is an iterative process that works on finding the mean between the examples and adding them to the cluster that has the least squared error [35]. This error can be calculated using distance formulas like Euclidean distance and Manhattan distance. Particularly, Euclidean distance is used to calculate the new means on which the new centroids are updated. This process is repeated until when there are no new assignments in the clusters.

Given a set of $\mathrm{n}$-dimensional samples $\left(i_{1}, i_{2}, \ldots, i_{n}\right)$ has been clustered into k-clusters $C_{1}, C_{2}, C_{3}, \ldots, C_{n}$. Each sample must be clustered into exactly one cluster. Initially means are randomly generated in the search space. The mean of each cluster is defined is by following equation:

$$
\operatorname{Mean}_{j}=\left(\frac{1}{n_{j}}\right) \sum \sum_{i=1}^{n_{j}} x_{i j}
$$


Where $x_{i} k$ is the $i^{t} h$ instance belonging to the $k^{t} h$ cluster in the search space. The centroid of each of the $k$ clusters becomes the newly made mean. Error within a cluster $C_{k}$ is calculated as explained in following equation:

$$
e_{k}^{2}=\sum_{i=1}^{n_{k}}\left(x_{i j}-M e a n_{j}\right)^{2}
$$

Where $e_{k}^{2}$ is the sum of error within the cluster. To calculate the sum of all the clusters following equation is used:

$$
E_{k}^{2}=\sum_{k=1}^{k} e_{k}^{2}
$$

Where $k$ is the $k_{t h}$ cluster in the search space and total error is sum of errors of the cluster in the search space.

- K-Nearest Neighbor : The k-nearest neighbor $(\mathrm{kNN})$ algorithm is a nonparametric technique used for both regression and classification [36. In case of classification, given a $\mathrm{n}$-dimensional input vector $\left(i_{1}, i_{2}, \ldots, i_{n}\right)$ with label $Y$ lie in space and and $\mathrm{p}$ be the total number of features $\left(f_{1}, f_{2}, . . f_{n}\right) . K$ is the number of nearest neighbors to which approximations are made with the test vector. An unlabeled example is assigned a labeled voted by nearest neighbors using ED as expressed in following equation7:

$$
\begin{aligned}
& d(x, y)=\sqrt{\sum_{i=1}^{n}\left(I_{i}(x)-I_{i}(y)\right)^{2}} \\
& y(x)=\arg \max _{y \in Y} \sum_{i=1}^{k} \delta\left(y, y\left(y_{i}\right)\right)
\end{aligned}
$$

Where $y_{i}$ is the nearest neighbor. and $\delta\left(y, y\left(y_{i}\right)=1\right.$ if $y=y\left(y_{i}\right)$ otherwise $\delta\left(y, y\left(y_{i}\right)=0\right.$. Labeled is assigned to the test instance on the basis of ED between them. There are many other metrics but ED is one of the most common metric.

\section{7 | Learning and Parameter Tuning}

Below we explain classification algorithms and their parameters.

- K-Means : K-means is an iterative algorithm that works on finding the mean between the examples and adding them to the cluster that has the least squared error. The most important parameter is distance function. In our case, we chose euclidean distance as it was performing well in our case as compared to Manhattan distance. All other parameters were kept as default.

- K-Nearest Neighbor (kNN) : An unlabeled example is assigned a labeled voted by nearest neighbors using Euclidean distance. Two most important parameters are distance function and number of nearest neighbor. We chose Euclidean distance as distance function and $\mathrm{K}=3$ nearest neighbors. We got the best overall performance of $99 \%$ using the above-described parameters.

The next section explains the Evaluation and Results. 


\section{4 | EVALUATION}

In this section, we explain our experiments, different performance measures for evaluation, present and discuss our results for the activity recognition. We perform different experimental analysis at different stages of the dataset to make it understandable for future use. We perform 10-fold cross-validation for evaluating the performance of the machine learning model.

\section{1 | Evaluation Measures}

Evaluation measures are a vital part to assess the performance of the classifiers. Almost all evaluation measures depend on the nature of the data. Mostly accuracy is taken as a basic measure but it is in the case when data is balanced. However, when the data is imbalanced, it does not provide reliable information. One can easily understand the performance of accuracy measures in case of data imbalance by seeing the confusion matrix. Below, we illustrate terms that can be useful for evaluation measure analysis.

Several measures are:

$$
\begin{gathered}
\text { recall }=\frac{T P}{T P+F N} \\
\text { precision }=\frac{T P}{T P+F P} \\
\text { accuracy }=\frac{T P}{N} \\
f-\text { score }=2 \times \frac{\text { precision } \times \text { recall }}{\text { precision }+ \text { recall }}
\end{gathered}
$$

For a fair comparison, we use the same evaluation measures as used in base papers.

$T P$ represents the true positive rate that is correctly classified instances, $F N$ represents a false negative rate that is wrongly recognized instances. $N$ represents total instances of all activities. $F P$ represents False Positive rate that is samples of other activities wrongly recognized as one activity samples. The recall is calculated by dividing $T P$ by $T P+F N$. Precision is calculated by $T P$ dividing by $T P+F P$. Accuracy is calculated by $T P$ divided by $N$. $\mathrm{f}$-score is computed as the harmonic mean of recall and precision.

\section{2 | Description of Experiments}

For experiments, we collect labeled data from smartphone sensors and then preprocessed data to remove noise as explained in Section 3.4 Difficult activities like upstairs, downstairs, fast running, and fast walking requires more human energy than other activities, thus, instances of these activities are fewer than other activities. The collected data of each participant for each activity is cleaned and grouped based on having complex inter-class variations and sharing common semantic information using k-means clustering. We make 8 clusters for 15 activities as explained in 
the section activity representation 3 after analyzing the similarity between activities. Moreover, we analyzed different cluster numbers. After clustering, we use the k-Nearest Neighbor classifier to recognize activity instances from 8 clusters.

\subsection{Activity Representation Analysis}

We chose 15 activities to recognize: standing, sitting, sitting cross leg, sitting left leg over right, sitting right leg over left, walking normal, walking fast, jogging, fast running, laying straight, Laying Over Right Side (Lateral Right Position), Laying Over Left Side (Lateral left Position, cycling, Upstairs and Downstairs. We select these activities because these activities are performed regularly by users. By analyzing data, we saw that some activities represent a similar pattern and some show distinct behavior and pattern.

Figure 3 and 4 plots the accelerometer, gyroscope and magnetometer data of the fifteen activities. It can be clearly seen that sitting activity in Subfigures $3 a|3 b 3 c| 3 d$, standing activity in $3 e$ and laying activity in Subfigures $3 f$ 3g $3 \mathrm{~h}$ do not depict periodic pattern but do have distinctive patterns, based on the relative magnitudes of the $x, y$, and $z$-axis values for each of the sensors, while the all other activities, which involve repetitive motions, do depict periodic pattern. For most activities, the values of the $y$-axis have the largest accelerations. This is due to Earth's gravitational pull, which causes the accelerometer to measure a value of $9.8 \mathrm{~m} / \mathrm{s} 2$ in the direction of the Earth's center.

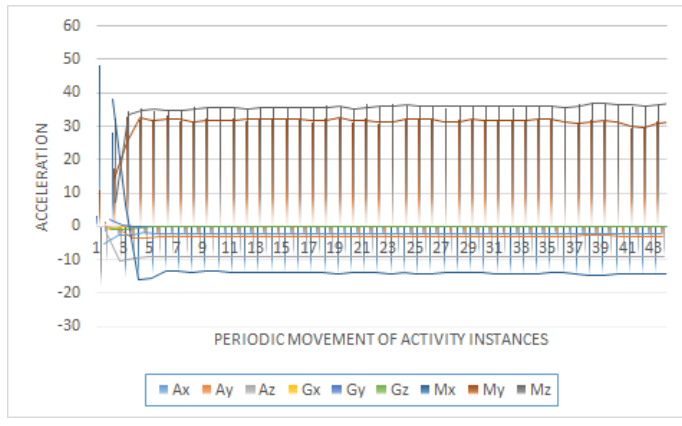

(a) Sitting Straight

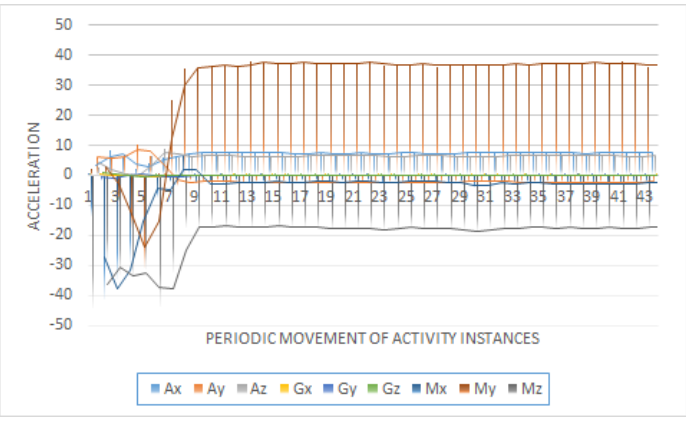

(b) Sitting Right Cross Leg

The periodic patterns for walking are shown in Subfigures 4a 4b], jogging in Subfigures 4c, 4d, ascending stairs descending stairs in Subfigure 4e 4f], and cycling are shown in Subfigure $4 \mathrm{~g}$ can be described in terms of the periodic peaks between each repetition of activity instance for each sensor and by the relative magnitudes of the acceleration values. The graph plot for walking shows a continuous series peaks along the $y$-axis and z-axis of the accelerometer. The distance between the peaks is also continuous. It can be observed that the repetitive activities represent a continuous peak to peak behavior.

Cycling, upstairs and downstairs in Subfigures $4 \mathrm{e}, 4 \mathrm{f}, 4 \mathrm{~g}$ shares similar repetitive behavior that is why the machine learning model confuses these activities with each other and same is the reason with walking and running, model confuses these with each other as well. 


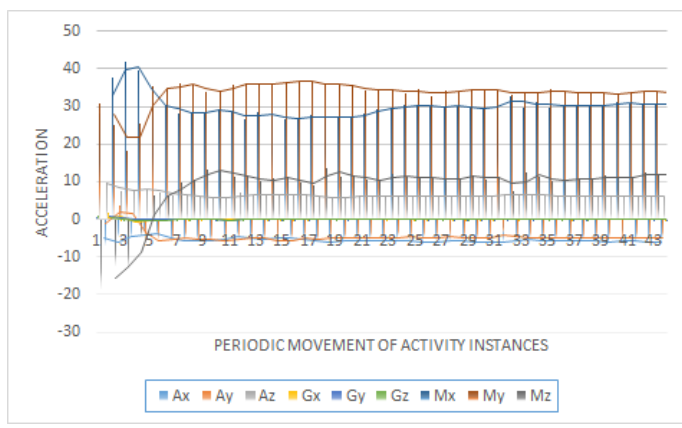

(c) Sitting Left Cross Leg

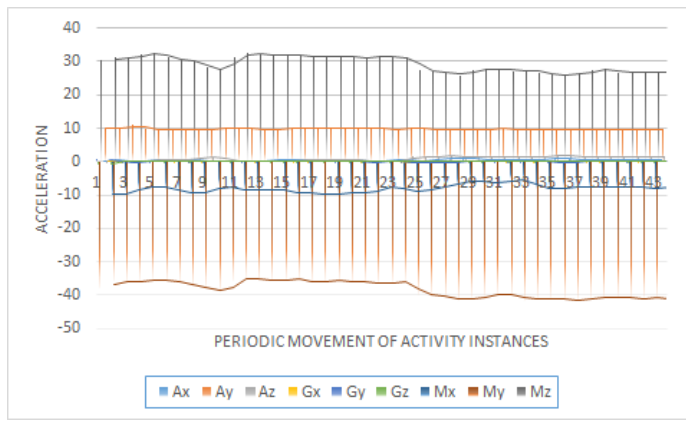

(e) Standing

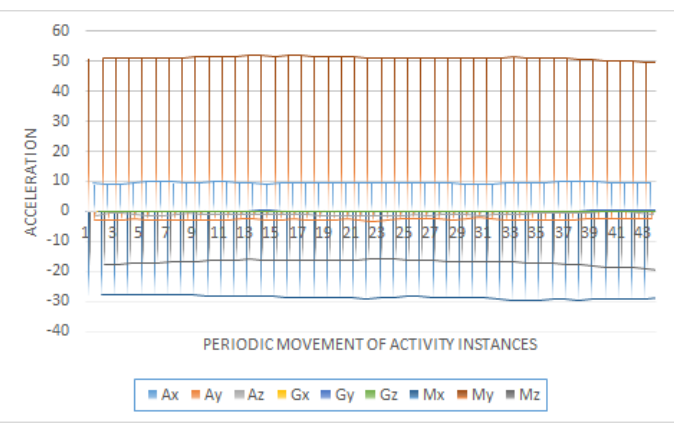

(g) Laying Over Left Side

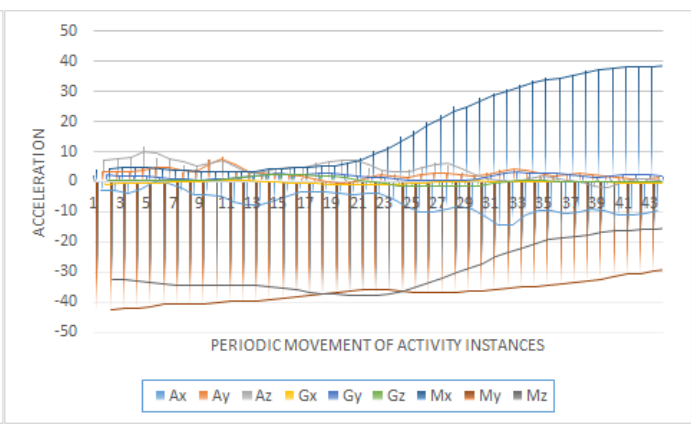

(d) Sitting Cross Legs

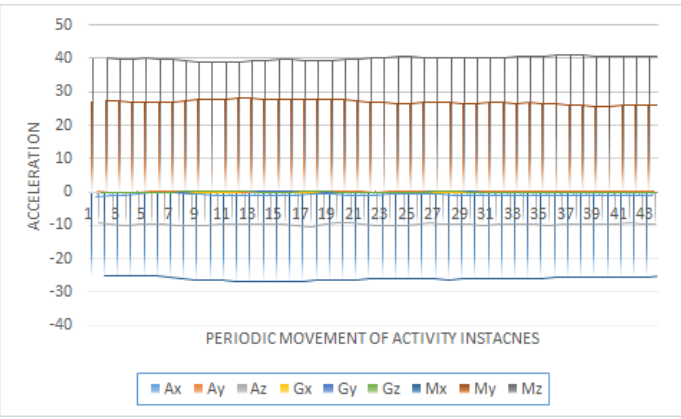

(f) Laying Straight

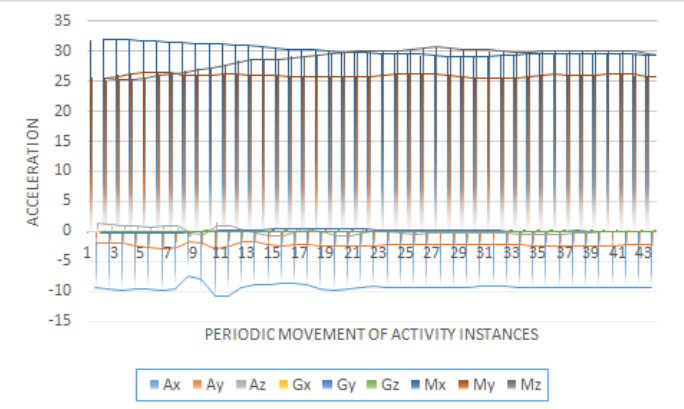

(h) Laying Over Right Side

FIGURE 3 Acceleration Plots for the Stable Physical Activities using Three Smartphone Sensors: Accelerometer, Gyroscope, and Magnetometer 


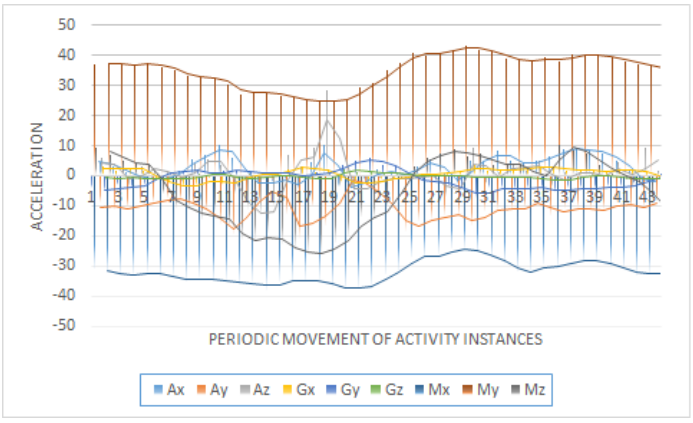

(a) Walking Normal
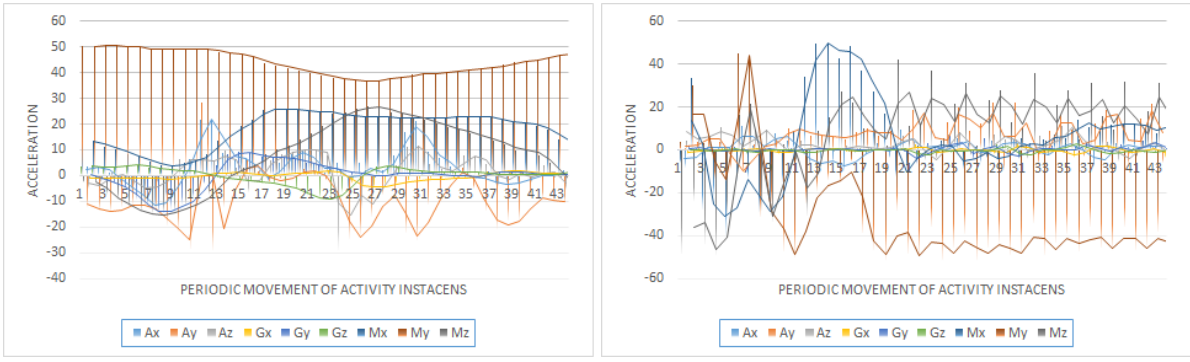

(b) Walking Fast

(c) Jogging
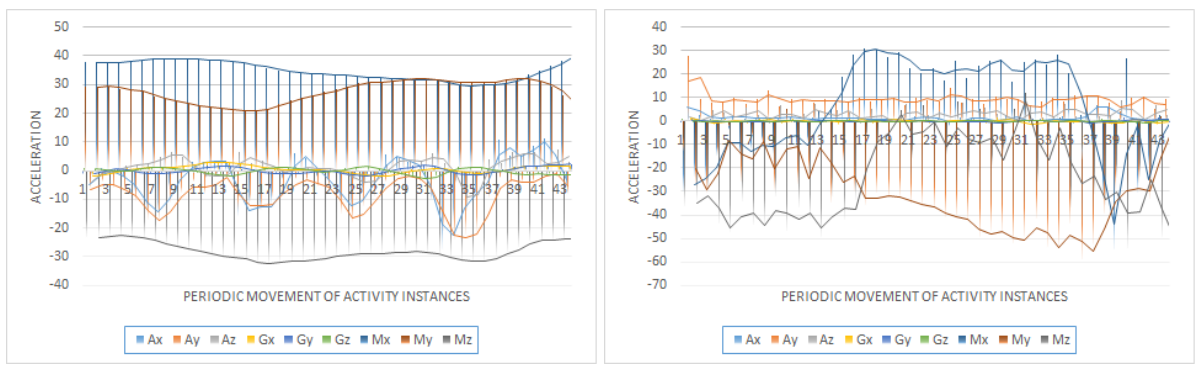

(d) Running Fast

(e) Upstairs

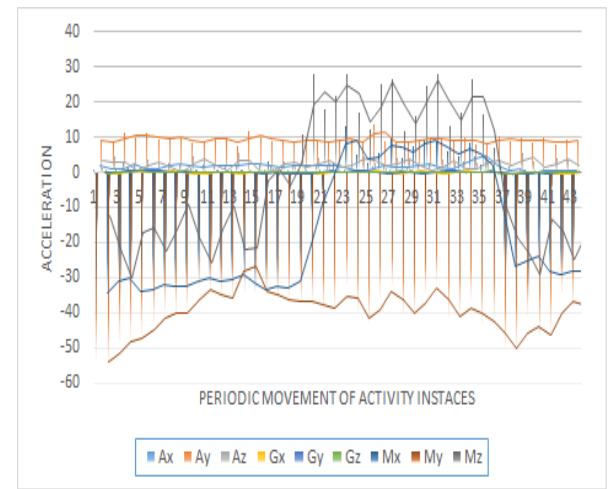

(f) Downstairs

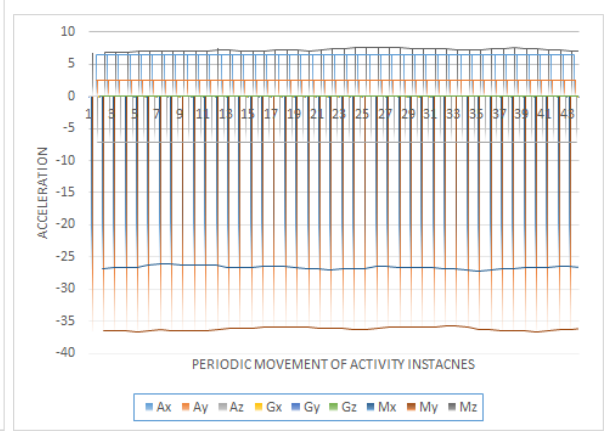

(g) Cycling

FIG URE 4 Acceleration Plots for the Periodic Physical Activities using Three Smartphone Sensors: Accelerometer, Gyroscope, and Magnetometer 


\section{4 | Results}

The detailed results of PARCIV are shown in Table 4 The Table shows the precision, recall, f-score and accuracy metric of each activity. PARCIV achieved the best accuracy for simple activities as well as activities having complex inter-class variations. PARCIV achieved $100 \%$ precision, recall, f-score and accuracy for activities 'Sitting Right Cross Leg', 'Cross Leg', 'Standing', 'Sitting Left Cross Leg', 'Straight Laying', 'Sitting Straight', 'Laying Over Left Side', 'Laying Over Right Side' and 'Cycling. PARCIV also achieved 98\% precision, recall, f-score and accuracy in case of activities having complex inter-class variations (i.e., Upstairs, Downstairs, Fast Running, Fast Walking, Jogging and Walking Normal). The most difficult activities to recognize have fewer inter-class variations and have fewer instances than others due to difficulties in performing these activities. The data balancing phase gives an advantage to this problem for increasing the variance. We achieved $99 \%$ precision, recall, f-score and accuracy on average for all 15 types of physical activities that show the effectiveness of PARCIV. The confusion matrix in Figure 5 ensure the reliability of PARCIV. This matrix illustrates how many examples of each activity often confused with the other activities. It shows that the maximum confusion is only $3 \%$ of 'Walking Normal' with the 'Fast Walking' activity. It also shows that all other activities are recognized efficiently with minimal confusion.

TAB LE 4 Results with Evaluation Measures of each Activity using PARCIV. The Precision, Recall and Accuracy are in Percentage and the F-score is in Range [0,1]

\begin{tabular}{|c|c|c|c|c|}
\hline Activities & Precision\% & Recall\% & f-Value & Accuracy\% \\
\hline Upstairs & 98.5 & 99.2 & 0.99 & 97.7 \\
\hline Sitting Right Cross Leg & 100.0 & 99.9 & 0.99 & 99.9 \\
\hline Fast Running & 98.6 & 98.03 & 0.98 & 96.7 \\
\hline Jogging & 98.25 & 96.9 & 0.98 & 95.3 \\
\hline Downstairs & 98.5 & 99.5 & 0.99 & 98.05 \\
\hline Cross Leg & 100.0 & 99.97 & 0.99 & 99.98 \\
\hline Standing & 100.0 & 99.7 & 0.99 & 99.7 \\
\hline Fast Walking & 96.03 & 98.03 & 0.97 & 94.2 \\
\hline Sitting Left Cross Leg & 100.0 & 99.9 & 0.99 & 99.9 \\
\hline Walking Normal & 95.8 & 96.3 & 0.96 & 92.4 \\
\hline Straight Laying & 100.0 & 99.98 & 0.99 & 99.98 \\
\hline Sitting Straight & 100.0 & 100.0 & 0.1 & 100.0 \\
\hline Laying over Left Side & 100.0 & 100.0 & 0.1 & 100.0 \\
\hline Laying over Right Side & 100.0 & 100.0 & 0.1 & 100.0 \\
\hline Cycling & 100.0 & 100.0 & 0.1 & 100.0 \\
\hline Avg. & 99.04 & 99.16 & 0.99 & 98.2 \\
\hline
\end{tabular}




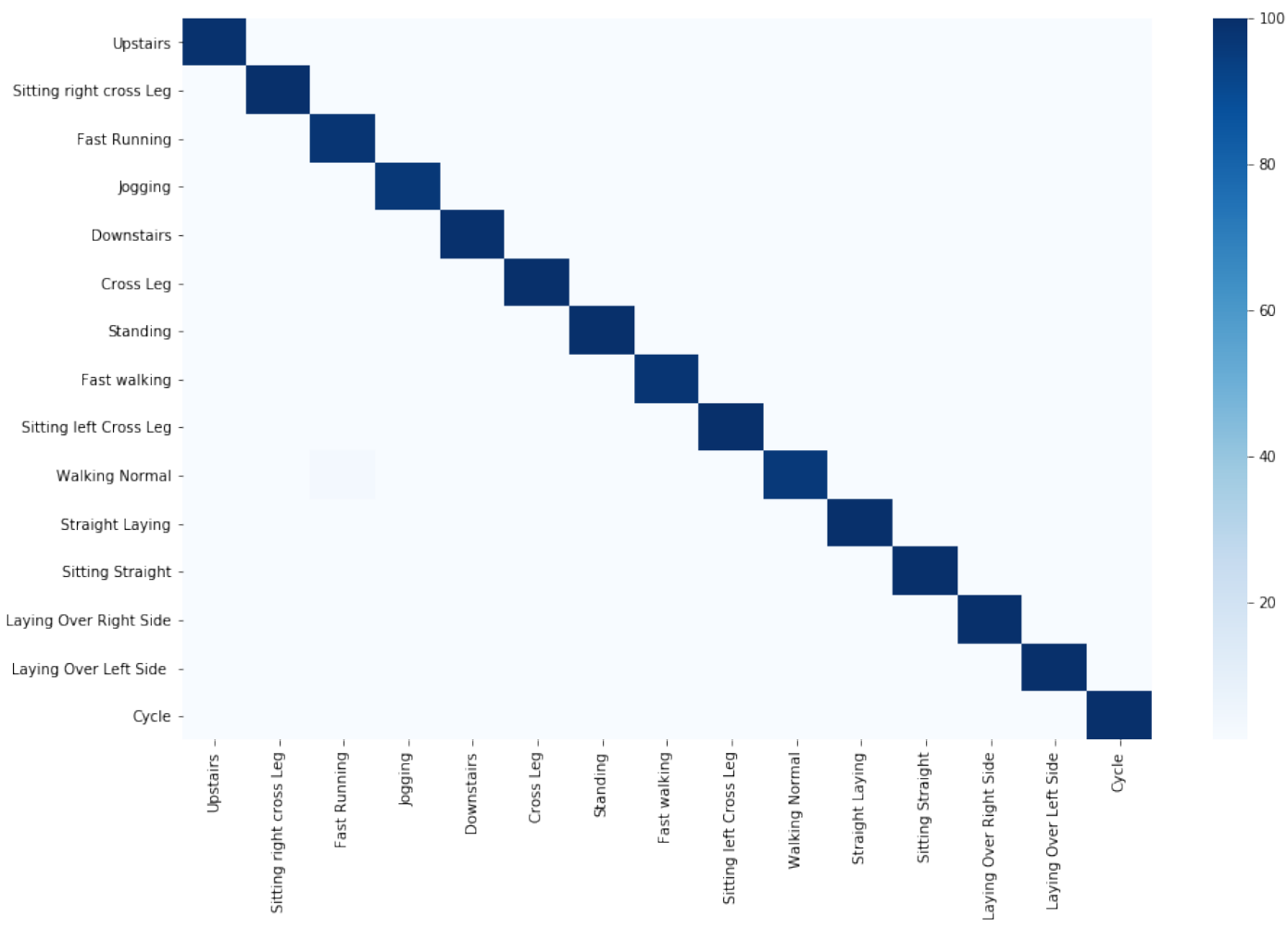

FIGURE 5 Confusion Matrix of PARCIV

\section{5 | Comparative Analysis}

We present overall performance comparison in Table 5 by using evaluation measures: precision, recall, $\mathrm{f}$-score and accuracy. The confusion matrices presents more detailed results for activities having complex inter-class variations. It shows the distribution of true positive, true negative, false negative and false positive instances concerning each activity. The previous studies on smartphone-based physical activity recognition reports the accuracy between $70.0 \%$ and $97.0 \%$. Accuracy is not a good measure when it comes to the different sample sizes of each activity class. In [2, authors claimed that their MCODE algorithm is more robust than $\mathrm{K}$-means but their results show $10 \%$ lower accuracy than our approach based on K-means. In [37, the authors show only the accuracy measure which is equivalent to our approach. They did not show other evaluation measures and confusion matrix to validate variations that exist between activities. The study [23], used a high computational cost algorithm CNN on dynamic features that even provides less accuracy than our approach. The study 24] also used a high computational cost algorithm DBN with 60 and 20 neurons in hidden layers and gained $3 \%$ less accuracy than our approach. The study [27], applied five classifiers: MLP, SVM, RF and Logit Boost (LG). They got the highest $90 \%$ accuracy with an ensemble method of MLP, LG, and SVM. In [21], authors used a self-collected dataset of five common activities and only consider accuracy which is $10 \%$ lower than our approach. Their confusion matrix shows that most activities were wrongly recognized as walking.

The confusion matrix in Figure 6 presents the comparison of PARCIV with state-of-the-art study [2]. It demonstrates that PARCIV provides more accurate results on three common activities having complex inter-class variations. 
It shows that PARCIV achieved 6\%, 14\%, and $2 \%$ higher score than [2] for walking, fast walking, and running activities respectively.

The bar graph in figure 7 presents the comparison results of proposed approach PARCIV with the study [20] and [16] using f-score on [16] dataset. Authors in [20] used ensemble method based on SVM. Its analysed that our proposed approach PARCIV outperforms of $16 \%, 17 \%, 20 \%$ and $12 \%$ than 20 on walking, jogging, upstairs and downstairs activities respectively. In comparison of [16, PARCIV gives $10 \%, 3 \%, 32 \%$ and $36 \%$ higher f-score on walking, jogging, upstairs and downstairs activities respectively.

TABLE 5 Comparison of PARCIV with State-of-the-art Approaches

\begin{tabular}{|c|c|c|c|c|c|c|c|}
\hline Approach & Dataset & Activities & Splitting & Precision & Recall & F-Score & Accuracy \\
\hline PARCIV & $\begin{array}{c}\text { Self } \\
\text { Collected }\end{array}$ & Table 3 & Ten-Fold & 99.04 & 99.16 & 0.99 & 98.2 \\
\hline PARCIV & 16] & $\begin{array}{l}\text { Walking, Jogging, Sitting, } \\
\text { Upstairs, Downstairs, Standing }\end{array}$ & Ten-Fold & 94.04 & 93.86 & 0.94 & 95.2 \\
\hline [2] & 16,38 & $\begin{array}{l}\text { Walking, Fast Walking, } \\
\text { Running }\end{array}$ & NA & 83.0 & 89.0 & 0.85 & 88.0 \\
\hline 37] & 39,37 & $\begin{array}{l}\text { Sitting, Standing, Laying, } \\
\text { Walking, Upstairs, Downstairs }\end{array}$ & $\begin{array}{l}\text { Train }=70 \% \\
\text { Test }=30 \%\end{array}$ & NA & NA & NA & $\begin{array}{l}97.3 \\
98.8\end{array}$ \\
\hline 23] & 39] & $\begin{array}{l}\text { Sitting, Standing, Laying, } \\
\text { Walking, Upstairs, Downstairs }\end{array}$ & $\begin{array}{l}\text { Train }=70 \% \\
\text { Test }=30 \%\end{array}$ & NA & NA & NA & 90.5 \\
\hline 24 & 391 & $\begin{array}{l}\text { Standing, Sitting, Lying, } \\
\text { Walking, Upstairs, Downstairs, } \\
\text { Stand-Sit, Sit-Stand, Sit-Lie, } \\
\text { Lie-Sit, Stand-Lie, Lie-Stand }\end{array}$ & $\begin{array}{l}\text { Train }=70 \% \\
\text { Test }=30 \%\end{array}$ & NA & NA & NA & 95.8 \\
\hline 18] & 18,39 & $\begin{array}{l}\text { Standing, Walking, Running, } \\
\text { Jumping,Cycling, Driving, } \\
\text { Upstairs, Downstairs. }\end{array}$ & NA & 96.9 & 96.9 & 96.9 & 99.2 \\
\hline 21] & 21] & $\begin{array}{l}\text { Static, Walking, Running, } \\
\text { Upstairs, Downstairs }\end{array}$ & Ten Fold & NA & NA & NA & 89.6 \\
\hline 8] & 8 & $\begin{array}{l}\text { Walk, Sit, Stand, Jogging, } \\
\text { Biking, Upstairs, Downstairs }\end{array}$ & Ten Fold & NA & NA & NA & 90.0 \\
\hline 27] & 27] & $\begin{array}{l}\text { Slow Walk, Fast Walk, Running, } \\
\text { Stairs-Up, Stairs-Down, Dancing }\end{array}$ & Ten Fold & NA & NA & NA & 90.0 \\
\hline 20] & 16 & $\begin{array}{l}\text { Walking, Jogging, Sitting, } \\
\text { Upstairs, Downstairs }\end{array}$ & $\begin{array}{l}\text { Train }=40 \%, \\
\text { Validate }=30 \% \\
\text { Test }=30 \%\end{array}$ & NA & NA & 82.0 & NA \\
\hline 16] & 16] & $\begin{array}{l}\text { Walking, Jogging, Sitting, } \\
\text { Upstairs, Downstairs, Standing }\end{array}$ & Ten Fold & NA & NA & 91.7 & NA \\
\hline
\end{tabular}




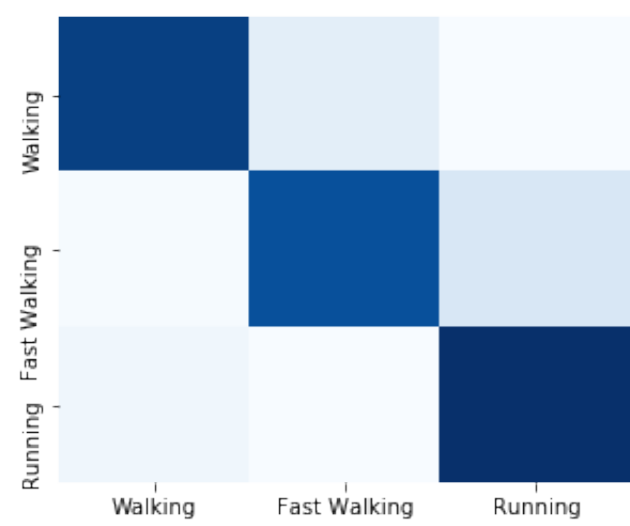

(a) Confusion matrix of the existing study $[2$
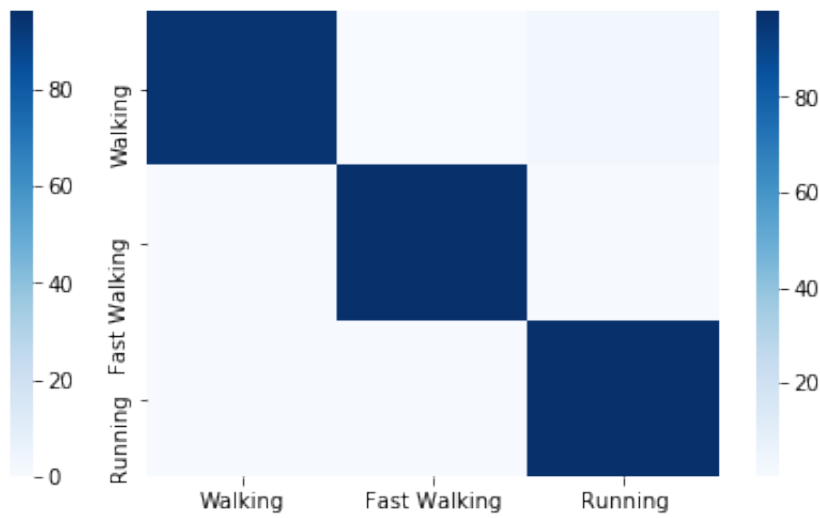

(b) Confusion matrix of PARCIV

FIGURE 6 Confusion Matrix Comparison with state-of-the-art

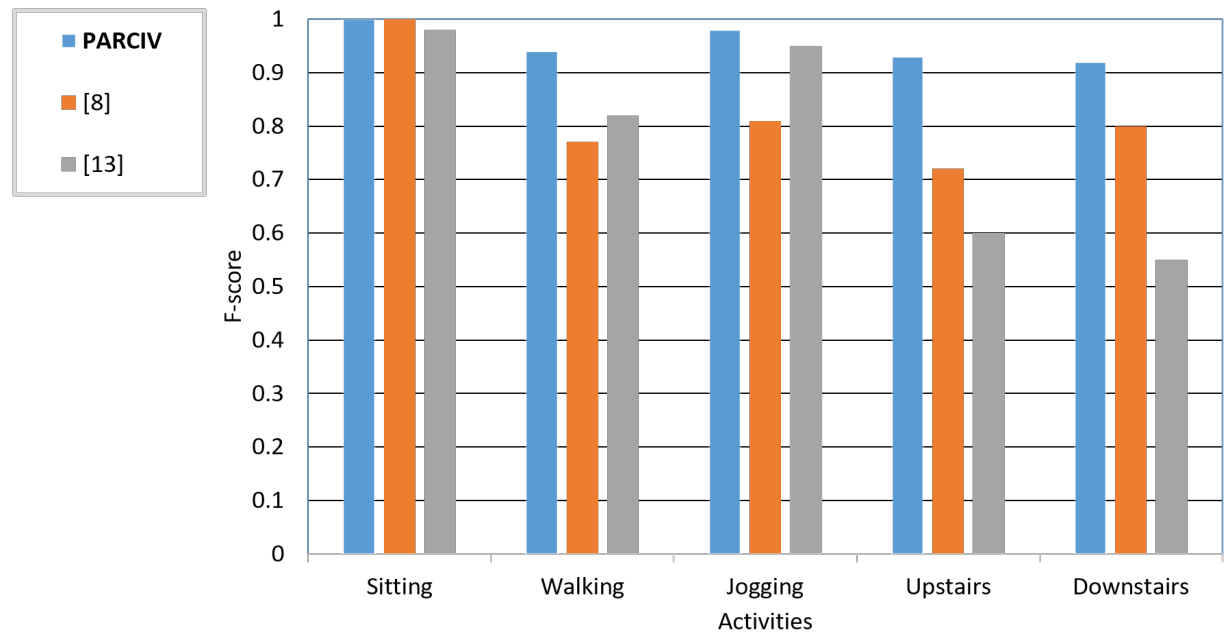

FIGURE 7 Bar graph Illustrating Comparison Results of PARCIV with the Existing Study [20] and [16] using f-score on 16 Dataset 


\section{6 | Discussion}

Table 4 shows the best performance of $98.2 \%$ of our model on a self-collected dataset having activities having complex inter-class variations (i.e., Upstairs, Downstairs, Walking Normal, Walking Fast and Laying positions). We also evaluate our approach on 16 dataset and shows the comparison with the studies that used the same dataset. As in figure 7 it shows that our proposed approach PARCIV outperforms than [16] and [20]. We generally achieve 96\%99\% accuracies for all activities including complex inter-class activities. The activities standing and sitting appears to be easier to recognize than other activities because of the still acceleration readings. It is analyzed that upstairs, downstairs, jogging, running and laying positions were more difficult to recognize because these similar activities have less inter-class variations since confused with each other. The Table 5 shows a quality comparison of PARCIV with

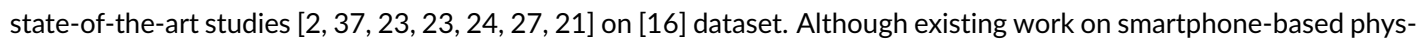
ical activity recognition shows an accuracy between $70.0 \%$ and $97.0 \%$. These variations can be due to: the dataset, the machine learning algorithm, hyper-parameter tuning, only used a specific set of activities and when the testing set is too small (i.e., 20\%).

\section{5 | CONCLUSION AND FUTURE WORK}

In this paper, a two-layered approach PARCIV is proposed that recognizes simple as well as activities having complex inter-class variations on a fine-grained level. PARCIV first group similar activities based on semantic data using the k-Means clustering algorithm and then recognize different similar activities using the kNN classification algorithm on a fine-grained level. We collected labeled data from smartphone sensors: accelerometer, gyroscope, and magnetometer for the evaluation of PARCIV. Ten participants were asked to performed activities while keeping the smartphone in the pocket. PARCIV achieved an accuracy of $96 \%-99 \%$ for the recognition of fifteen activities on the self-collected dataset and $95.2 \%$ for the recognition of six activities on [16] dataset. Our experimental results show that the proposed method is practical and capable of increasing the recognition rate of normal physical activities as well as complex activities having complex inter-class variations. Moreover, it is shown that our proposed approach provides almost a $15 \%$ higher recognition rate than the state-of-art methods, primarily for activities having complex inter-class variations (e.g., walking normal, fast walking, upstairs, downstairs, laying positions).

In the future, we intend to recognize more complex physical activities. we plan to enhance the recognition process in the following way: 1) using deep learning methods to extract patterns for overlapping activities 2) gathering data from many users which belong to different age groups including old ages 3) gathering data from cognitively impaired individuals to correlate their health with physical activity patterns.

\section{conflict of interest}

The authors declare no potential conflict of interest.

\section{references}

[1] Sarwar MU, Javed AR. Collaborative Health Care Plan through Crowdsource Data using Ambient Application. In: 2019 22nd International Multitopic Conference (INMIC) IEEE; 2019. p. 1-6.

[2] Lu Y, Wei Y, Liu L, Zhong J, Sun L, Liu Y. Towards unsupervised physical activity recognition using smartphone accelerometers. Multimedia Tools and Applications 2017;76(8):10701-10719. 
[3] Kutia S, Chauhdary SH, Iwendi C, Liu L, Yong W, Bashir AK. Socio-Technological factors affecting user's adoption of eHealth functionalities: A case study of China and Ukraine eHealth systems. IEEE Access 2019;7:90777-90788.

[4] Sultan S, Javed A, Irtaza A, Dawood H, Dawood H, Bashir AK. A hybrid egocentric video summarization method to improve the healthcare for Alzheimer patients. Journal of Ambient Intelligence and Humanized Computing 2019;10(10):4197-4206.

[5] Warburton DE, Nicol CW, Bredin SS. Health benefits of physical activity: the evidence. Cmaj 2006;174(6):801-809.

[6] Alazab RM, Almohsen ARM. Obesity indices as a risk factor of skin diseases: A Case-control study conducted in Cairo, Egypt. South East Asia Journal of Public Health 2015;5(2):23-29.

[7] Bao L, Intille SS. Activity recognition from user-annotated acceleration data. In: International conference on pervasive computing Springer; 2004. p. 1-17.

[8] Shoaib M, Bosch S, Incel OD, Scholten H, Havinga PJ. Fusion of smartphone motion sensors for physical activity recognition. Sensors 2014;14(6):10146-10176.

[9] Maestre-Rendon JR, Rivera-Roman TA, Fernandez-Jaramillo AA, Guerrón Paredes NE, Serrano Olmedo JJ. A NonContact Photoplethysmography Technique for the Estimation of Heart Rate via Smartphone. Applied Sciences 2020;10(1):154.

[10] Su X, Tong H, Ji P. Activity recognition with smartphone sensors. Tsinghua science and technology 2014;19(3):235-249.

[11] Pei L, Guinness R, Chen R, Liu J, Kuusniemi H, Chen Y, et al. Human behavior cognition using smartphone sensors. Sensors 2013;13(2):1402-1424.

[12] Alazab M, Tang M. Deep Learning Applications for Cyber Security. Springer; 2019.

[13] Fahim M, Baker T, Khattak AM, Shah B, Aleem S, Chow F. Context mining of sedentary behaviour for promoting selfawareness using a smartphone. Sensors 2018;18(3):874.

[14] Baker T, Mackay M, Randles M, Taleb-Bendiab A. Intention-oriented programming support for runtime adaptive autonomic cloud-based applications. Computers \& Electrical Engineering 2013;39(7):2400-2412.

[15] Fahim M, Baker T, Khattak AM, Alfandi O. Alert me: Enhancing active lifestyle via observing sedentary behavior using mobile sensing systems. In: 2017 IEEE 19th International Conference on e-Health Networking, Applications and Services (Healthcom) IEEE; 2017. p. 1-4.

[16] Kwapisz JR, Weiss GM, Moore SA. Activity recognition using cell phone accelerometers. ACM SigKDD Explorations Newsletter 2011;12(2):74-82.

[17] Yan Z, Subbaraju V, Chakraborty D, Misra A, Aberer K. Energy-efficient continuous activity recognition on mobile phones: An activity-adaptive approach. In: 2012 16th international symposium on wearable computers leee; 2012. p. 17-24.

[18] Morillo LMS, Gonzalez-Abril L, Ramirez JAO, la Concepcion D, Alvarez MA. Low energy physical activity recognition system on smartphones. Sensors 2015;15(3):5163-5196.

[19] Srinivasan $V$, Phan T. An accurate two-tier classifier for efficient duty-cycling of smartphone activity recognition systems. In: Proceedings of the Third International Workshop on Sensing Applications on Mobile Phones; 2012. p. 1-5.

[20] Zheng Y, Wong WK, Guan X, Trost S. Physical activity recognition from accelerometer data using a multi-scale ensemble method. In: Twenty-Fifth IAAI Conference; 2013.

[21] Miao F, He Y, Liu J, Li Y, Ayoola I. Identifying typical physical activity on smartphone with varying positions and orientations. Biomedical engineering online 2015;14(1):32. 
[22] Chen Z, Zhu Q, Soh YC, Zhang L. Robust human activity recognition using smartphone sensors via CT-PCA and online SVM. IEEE Transactions on Industrial Informatics 2017;13(6):3070-3080.

[23] Nakano K, Chakraborty B. Effect of dynamic feature for human activity recognition using smartphone sensors. In: 2017 IEEE 8th International Conference on Awareness Science and Technology (iCAST) IEEE; 2017. p. 539-543.

[24] Hassan MM, Uddin MZ, Mohamed A, Almogren A. A robust human activity recognition system using smartphone sensors and deep learning. Future Generation Computer Systems 2018;81:307-313.

[25] Wannenburg J, Malekian R. Physical activity recognition from smartphone accelerometer data for user context awareness sensing. IEEE Transactions on Systems, Man, and Cybernetics: Systems 2016;47(12):3142-3149.

[26] de la Concepción MÁÁ, Morillo LMS, García JAÁ, González-Abril L. Mobile activity recognition and fall detection system for elderly people using Ameva algorithm. Pervasive and Mobile Computing 2017;34:3-13.

[27] Bayat A, Pomplun M, Tran DA. A study on human activity recognition using accelerometer data from smartphones. Procedia Computer Science 2014;34:450-457.

[28] Mannini A, Sabatini AM. Machine learning methods for classifying human physical activity from on-body accelerometers. Sensors 2010;10(2):1154-1175.

[29] Tapia EM, Intille SS, Haskell W, Larson K, Wright J, King A, et al. Real-time recognition of physical activities and their intensities using wireless accelerometers and a heart rate monitor. In: 2007 11th IEEE international symposium on wearable computers IEEE; 2007. p. 37-40.

[30] Gjoreski H, Kozina S, Gams M, Luštrek M. RAReFall-Real-time activity recognition and fall detection system. In: 2014 IEEE International Conference on Pervasive Computing and Communication Workshops (PERCOM WORKSHOPS) IEEE; 2014. p. 145-147.

[31] Rubaiyeat HA, Kim TS, Hasan MK. Real-time recognition of daily human activities using a single tri-axial accelerometer. In: 2010 5th International Conference on Embedded and Multimedia Computing IEEE; 2010. p. 1-5.

[32] Farhan AA, Yue C, Morillo R, Ware S, Lu J, Bi J, et al. Behavior vs. introspection: refining prediction of clinical depression via smartphone sensing data. In: 2016 IEEE Wireless Health (WH) IEEE; 2016. p. 1-8.

[33] Chawla NV, Bowyer KW, Hall LO, Kegelmeyer WP. SMOTE: synthetic minority over-sampling technique. Journal of artificial intelligence research 2002;16:321-357.

[34] Javed AR, Beg MO, Asim M, Baker T, Al-Bayatti AH. AlphaLogger: detecting motion-based side-channel attack using smartphone keystrokes. Journal of Ambient Intelligence and Humanized Computing 2020;p. 1-14.

[35] Hamerly G, Drake J. Accelerating Lloyd's algorithm for k-means clustering. In: Partitional clustering algorithms Springer; 2015.p. 41-78.

[36] Altman NS. An introduction to kernel and nearest-neighbor nonparametric regression. The American Statistician 1992;46(3):175-185.

[37] Chen Z, Jiang C, Xie L. A novel ensemble ELM for human activity recognition using smartphone sensors. IEEE Transactions on Industrial Informatics 2018;15(5):2691-2699.

[38] Wei Y, Liu L, Zhong J, Lu Y, Sun L. Unsupervised race walking recognition using smartphone accelerometers. In: International Conference on Knowledge Science, Engineering and Management Springer; 2015. p. 691-702.

[39] Anguita D, Ghio A, Oneto L, Parra X, Reyes-Ortiz JL. A public domain dataset for human activity recognition using smartphones. In: Esann; 2013. . 


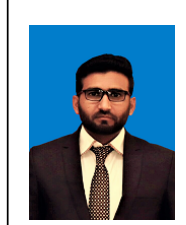

Muhammad Usman Sarwar is a lecturer at the Department of Computer Science, Air University, Islamabad, Pakistan. He has more than two years of teaching experience and three years of experience in R\&D projects. He published scientific articles in emerging technologies in international high ranked journals/proceedings. He got his Masters degree in Computer Science from National University of Computer and Emerging Sciences, Islamabad, Pakistan. His current research interests include mobile computing, data analysis, ambient intelligence, machine learning, promoting independent living and automated assessment of smart home residents, multi-agent healthcare framework using ambient intelligence, activity recognition and student life assessment.

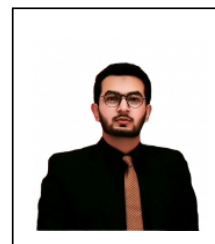

Abdul Rehman Javed is a research assistant at National Center for Cyber Security (NCCS), Air University, Islamabad, Pakistan. He received his Master's degree in Computer Science from National University of Computer and Emerging Sciences, Islamabad, Pakistan. His research interests include but are not limited to mobile and ubiquitous computing, data analysis, knowledge discovery, data mining, natural language processing, smart homes, and their applications in human activity analysis, human motion analysis and e-health. He aims to contribute to interdisciplinary research of computer science and human-related disciplines.

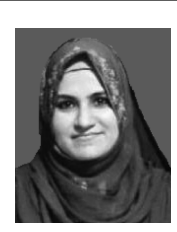

Farzana Kulsoom received the B.Sc. degree in computer engineering and the master's degree in telecommunication engineering from the University of Engineering and Technology, Taxila, Pakistan, in 2006 and 2012, respectively. She was a Research Associate with the Center of Excellence for ASIC Design and DSP and participated in several industrial projects and research activities. She is currently pursuing the Ph.D. degree with the University of Pavia, Italy, in the areas of signal processing and wireless communication. Her current research interests are MIMO-based communication, nextgeneration communication systems, and machine learning applied to signal processing.

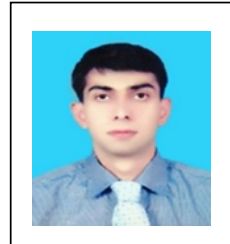

Suleman Khan received his Master's degree from the Department of Computer Science at Air University Islamabad, in 2019. He is currently a Research Associate at Air University, Pakistan. His research interests include network security, machine learning, and data science.

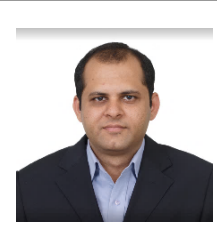

Usman Tariq is a skilled research engineer with a doctorate in Information and Communication Technology in Computer Science from Ajou University, S. Korea. Strong background in ad hoc networks and network communications. Experienced in managing and developing projects from conception to completion. I have worked in large international scale and long-term projects with multinational organizations. Currently, he is attached to Prince Sattam bin Abdul-Aziz Uni- 
versity as an associate professor in the College of Computer Engineering and Science. Usman's research interests span networking and security fields. His current research is focused on several network security problems: botnets, denial-of-service attacks, and IP spoofing. Additionally, he is interested in methodologies for conducting security.

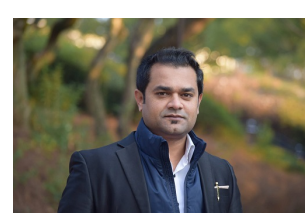

Ali Kashif Bashir is a Senior Lecturer at the Department of Computing and Mathematics, Manchester Metropolitan University, United Kingdom. He is also affiliated with SEECS, National University of Science and Technology, Pakistan as an Adjunct Professor. He is a senior member of IEEE, invited member of the IEEE Industrial Electronics Society, member of ACM, and Distinguished Speaker of ACM. His past assignments include Associate Professor of ICT, University of the Faroe Islands, Denmark; Osaka University, Japan; Nara National College of Technology, Japan; the National Fusion Research Institute, South Korea; Southern Power Company Ltd., South Korea, and the Seoul Metropolitan Government, South Korea. He has worked on several research and industrial projects of South Korean, Japanese and European agencies and Government Ministries. He is also advising several start-ups in the field of STEM-based education, blockchain, robotics, and smart homes. He received his Ph.D. in computer science and engineering from Korea University South Korea. He has authored over 100 research articles and is supervising/co-supervising several graduate (MS and Ph.D.) students. His research interests include the internet of things, wireless networks, distributed systems, network/cybersecurity, cloud/network function virtualization, etc. He is serving as the Editor-in-chief of the IEEE FUTURE DIRECTIONS NEWSLETTER. 